\title{
MRI Brain in Perinatal Hypoxia - A Case Series.
}

\author{
Dr. Sanjay Mhalasakant Khaladkar ${ }^{1}$, Dr. Aditi M.Gujarathi ${ }^{2}$, Dr. Vigyat Kamal ${ }^{3}$, \\ Dr. Anubhav Kamal ${ }^{4}$, Dr. Rajesh Kuber ${ }^{5}$ \\ ${ }^{1}$ Professor, Department of Radiology, ${ }^{2}$ Senior Resident, Department of Radiology, \\ ${ }^{3} P G$ Student, Department of Radiology, ${ }^{4}$ Senior Resident, Department of Radiology, \\ ${ }^{5}$ Professor, Department of Radiology, \& Dr. DY Patil Medical College \& Research Center, Pimpri, Pune
}

\begin{abstract}
:
Background: HIE is one of the most common cause of neonatal deaths and morbidity in children. Due to limited facilities in India, many a times HIE goes undetected only to present in later stages of life as handicapped children.

Objective: To study the Magnetic Resonance Imaging (MRI) brain findings in children having suffered perinatal hypoxia, to analyze MRI findings in brain based on time of perinatal hypoxia.

Material and Methods: A prospective Study was conducted on 100 patients suffered with perinatal hypoxia on Siemens 1.5 Tesla MAGNATOM AVANTO machine. Patterns of hypoxic ischemic brain injury in preterm and term infants of were evaluated.

Results: Out of 100 cases, $72 \%$ were abnormal and $28 \%$ were normal studies. $36 \%$ patients were preterms and $64 \%$ had term delivery. In patients with term delivery, $28.1 \%$ were normal and $71.8 \%$ were abnormal. In patients with preterm delivery, $72.3 \%$ cases were found to be abnormal while $27.7 \%$ cases did not show any significant abnormality.

Conclusion: MRI was a definitive diagnostic modality in perinatal hypoxia. Patterns of brain injury were determined by nature, timing and severity of insult. Imaging appearances were influenced by sequences used and time from injury.
\end{abstract}

\section{Introduction}

Hypoxic Ischemic Encephalopathy (HIE) refers to a combination of hypoxia, hypercarbia and metabolic acidosis as a consequence of occlusion of umbilical vessels or interference with placental perfusion in fetal life and/or due to lack of effective breathing after birth. Hypoxia literally means decreased oxygen supply and ischemia is a resultant damage to the tissues. Thus any cause which will lead to lack of oxygen supply to the brain tissues which is extremely necessary for the normal functioning of the brain and thus in turn the other body system; it will give rise to a series of events that will lead to injury to the brain tissues and certain clinical manifestations. Almost $40 \%$ of deaths under age 5 years in children occur in the neonatal period. Reported global totals of neonatal deaths due to non-specific conditions of HIE vary from 0.7 million to 1.6 million per year. Mortality rate in children with severe hypoxia is $25-50 \%$. Incidence of prenatal asphyxia is approximately $3.3 \%$ in India. It is usually related to gestational age and birth weight. ${ }^{[1]}$ Thus, outcomes of HIE are dangerous and permanent, making it a major burden for the patient, family and the society. Thus, it is very important to identify, evaluate and analyze the effects of HIE on the brain so as to develop better therapeutic strategies to decrease the brain injury in newborns and provide them a better future.

\section{Materials and Methods}

A prospective study was conducted on 100 patients in the department of Radio-diagnosis, Padmashree Dr. D.Y Patil Medical College from July 2012 over a period of 2years to September 2014. The study included all the patients of paediatric age group (birth to12 years of age) referred for MRI having suffered from perinatal hypoxia. It included neonates with clinical signs of bradycardia, hypotonia and who gave poor response to stimulation. In children; developmental delay, cerebral palsy, seizures, hemiplegia/paraplegia/quadriplegia were the criteria used for performing the MRI. Patients who were on MRI incompatible life support were excluded. Patients were evaluated by Siemens 1.5 Tesla MAGNATOM AVANTO machine. The bore size was $60 \mathrm{~cm}$ and the overall length was $160 \mathrm{~cm}$. The MRI system used zero Helium boil off technology. A $32 \mathrm{channel}$ head coil with iPAT compatibility was used. The proforma was designed on the objective of the study and it was used after modification.A structured proforma was used to note the detailed clinical history, birth history, developmental history, immunization details, anthropometry, physical examination findings, systemic examination findings and investigation findings.Patient was positioned in the supine position and a head coil was placed around the head to obtain a uniform signal to noise ratio. MRI examination of the brain was performed in axial, sagittal and coronal planes. A Sagittal acquisition through the mid Sagittal plane was used as 
an initial localizer for subsequent axial and coronal planes. T1 (TR/ TE, 210-710/6-14 ms, flip angle 90 ${ }^{\circ}$,

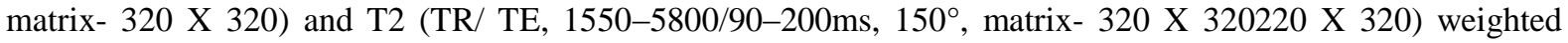
images were obtained. Fluid Attenuation Inversion recovery (FLAIR) (TR/ TE, 8050/120 -130/1860-2000ms, flip angle $-150^{\circ}$, matrix- 256 X 320), Diffusion Weighted, Gradient Echo and IR sequences were also performed. ADC maps of brain were obtained in axial planes. DWI was done by using single-shot spin-echo echo-planar sequences (TR/TE, 5130-5000/74-68 ms with a b-value of 800-1000 s/mm2, matrix- 190 X 190). The conventional MR brain protocol included T1-weighted sequences, T2-weighted sequences and Gradient Echo (GRE) (TR/ TE, 700/26, flip angle-20, matrix- 132 X 192) sequences. Field of view of $230 \mathrm{~mm}$ and slice thickness of $5 \mathrm{~mm}$ for routine and $3 \mathrm{~mm}$ for IR sequences was used. T1-weighted and inversion recovery sequences provided excellent anatomic information as well as high contrast between gray and white matter. T2weighted sequences provided good contrast resolution between gray matter, unmyelinated white matter and myelinated white matter. Gradient Echo (GRE) sequences provided increased sensitivity for the detection of T2weighted magnetic susceptibility. Three-dimensional T1 IR sequence (3DT1 IR) (TR/TE, 4000-4120/70-74ms, flip angle- $150^{\circ}$,matrix- $154 \mathrm{X}$ 192) and allows thin $1 \mathrm{~mm}$ contiguous slices which can be reconstructed in any anatomic plane. T1-weighted IR sequence is helpful as it gives excellent images of brain anatomy and maturation. It helps in differentiating myelinated and unmyelinated WM.

\section{Observations and Results}

In our study of 100 cases, the lesions which were accurately diagnosed by MRI included pathologies such as germinal matrix hemorrhage, periventricular leukomalacia, cystic encephalomalacia, cerebral and cerebellar atrophy, corpus callosal thinning and agenesis and delayed myelination. Thus, in diagnosis of these conditions MRI was a definitive diagnostic modality. MRI was able to diagnose these conditions in $72 \%$ of the patients. However, in rest of the $28 \%$ patients, MRI was normal, even though the patient had a history of perinatal hypoxia or was symptomatic.

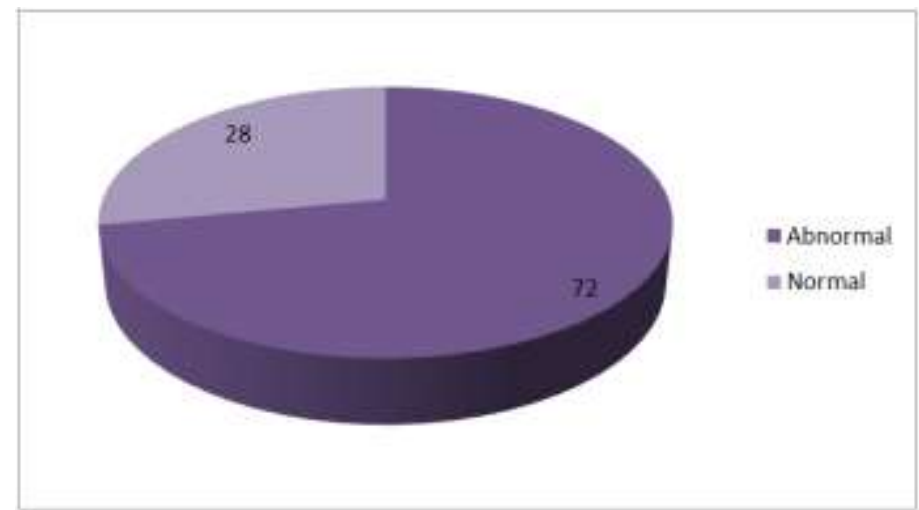

Figure-1 Distribution of total number of cases studied

\section{Sex Wise Distribution}

In this study MRI of the brain was done in 100 patients out of whom 56 were males and 46 were females as seen in the chart below. Thus, males were more commonly affected than females.

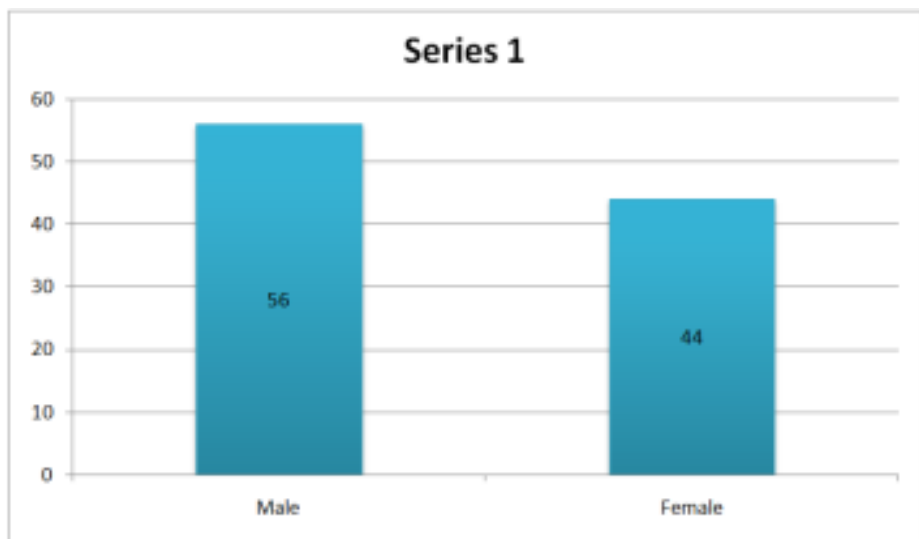

Figure-2:Sex distribution of children suffered with perinatal hypoxia. 


\section{Age Wise Distribution}

The age of patients ranged from 0 years to 12 years. It is clear from the table below that maximum pathologies were seen in patients who were in the age group of 1 to 5 years.

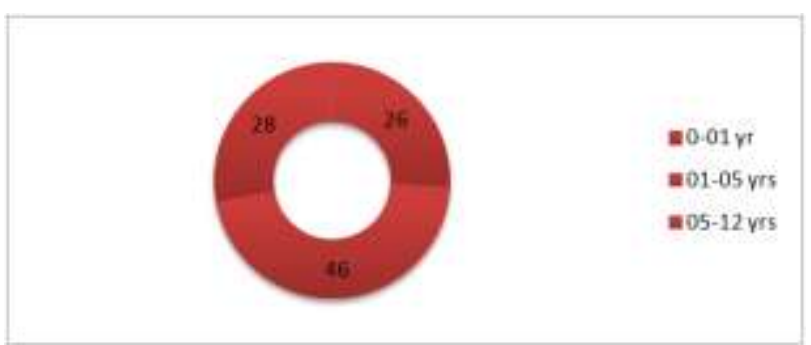

Figure-3 Age wise distribution of children suffered with perinatal hypoxia.

\section{Gestational Age Wise Distribution}

In our case study, 36 patients had a history of preterm delivery and 64 patients were term neonates.

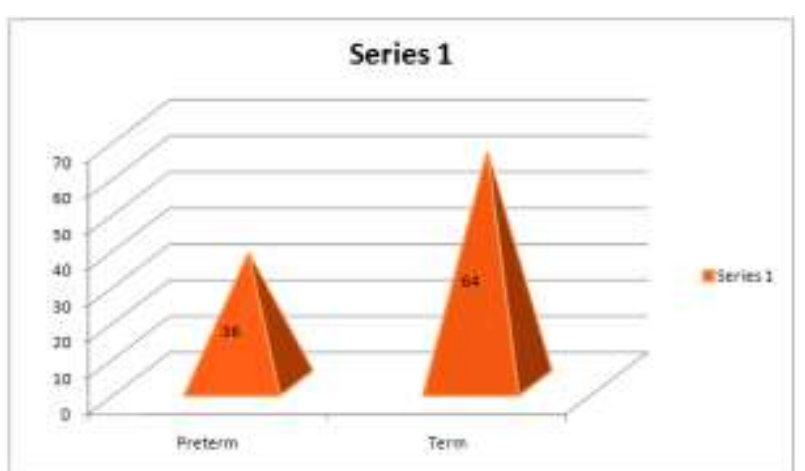

Figure - 4 Distribution of cases according to gestational age.

\section{Distribution of Risk Factors for HIE}

There are various risk factors for HIE in a preterm or term neonate. Amongst all maternal and fetal factors no specific cause was found in around $25 \%$ of the cases. It was followed by pre eclampsia and anemia. The other causes have been charted below.

\begin{tabular}{|l|l|l|}
\hline Risk Factors & Number & Percentage (n =100) \\
\hline Indiopathic & 25 & $25 \%$ \\
\hline Pre eclampsia & 20 & $20 \%$ \\
\hline Anemia & 15 & $15 \%$ \\
\hline Placental Factors & 10 & $10 \%$ \\
\hline Perinatal Infection & 8 & $8 \%$ \\
\hline Assisted Delivery & 7 & $7 \%$ \\
\hline Caesarean Section & 15 & $15 \%$ \\
\hline
\end{tabular}

Table -1 Distribution of risk factors for HIE.

\section{Distribution Of Cases According To The Gravid Status Of The Mothers}

Information of gravid status, antenatal examination, hospital or home delivery was obtained of all the mothers of 100 patients.

\begin{tabular}{|l|l|}
\hline Gravid status & Distribution $(\mathbf{n}=\mathbf{1 0 0})$ \\
\hline Primigravida & $55(55 \%)$ \\
\hline No antenatal examination & $44(80 \%)$ \\
\hline Undergone antenatal examination & $11(20 \%)$ \\
\hline Home deliveries & $15(27.2 \%)$ \\
\hline Hospital deliveries & $40(72.8 \%)$ \\
\hline Multigravida & $45(45 \%)$ \\
\hline No antenatal examination & $33(75 \%)$ \\
\hline Undergone antenatal examination & $12(25 \%)$ \\
\hline Home deliveries & $14(31.1 \%)$ \\
\hline Hospital deliveries & $31(68.9 \%)$ \\
\hline
\end{tabular}

Table-2 Distribution of Cases According To the Gravid Status ofthe Mothers. 


\section{Imaging Findings in Term Neonates}

64 cases of term neonates who suffered perinatal hypoxia underwent MRI, out of which 46 cases were abnormal (71.8\%). Of them, 36 showed white matter (cortical and/or periventricular) T2 hyperintensities, which was the most common abnormality found. Other abnormalities were cerebral atrophy, cystic encephalomalacia, delayed myelination, corpus callosum thinning, cerebellar atrophy, acute infarcts. 18 cases were normal (28.1 $\%)$.

\begin{tabular}{|l|l|l|}
\hline Findings & Number & Percentage (n =64) \\
\hline White matter hyperintensities & 36 & $60 \%$ \\
\hline Cerebral atrophy & 32 & $50 \%$ \\
\hline Delayed myelination & 20 & $31.2 \%$ \\
\hline Corpus callosum thinning & 14 & $21.8 \%$ \\
\hline Cystic encephalomalacia & 24 & $37.5 \%$ \\
\hline Acute infarcts & 10 & $15.6 \%$ \\
\hline Cerebellar atrophy & 10 & $15.6 \%$ \\
\hline Normal & 18 & $28.1 \%$ \\
\hline
\end{tabular}

Table-3MRI findings in HIE in term neonates.

\section{Imaging Findings In Preterm Neonates}

36 cases of preterm neonates who suffered perinatal hypoxia were evaluated in our study by MRI, out of which 10 were normal $(27.7 \%)$ and 26 were abnormal $(72.3 \%)$. Of the abnormal studies, the most common were periventricular leucomalacia (12) and cerebral atrophy (12).

Other pathologies which were observed were delayed myelination, corpus callosum thinning, cystic encephalomalacia, acute infarcts and cerebellar atrophy.

\begin{tabular}{|l|l|l|}
\hline Findings & Number & Percentage $(\mathbf{n}=\mathbf{3 6})$ \\
\hline Periventricular leukomalacia & 12 & $33.3 \%$ \\
\hline Cerebral atrophy & 12 & $33.3 \%$ \\
\hline Delayed myelination & 8 & $22.2 \%$ \\
\hline Corpus callosum thinning & 8 & $22.2 \%$ \\
\hline Cystic encephalomalacia & 8 & $22.2 \%$ \\
\hline Acute infarcts & 4 & $11.1 \%$ \\
\hline Cerebellar atrophy & 2 & $5.5 \%$ \\
\hline Germinal Matrix Hemorrhage & 2 & $5.5 \%$ \\
\hline Normal & 10 & $27.7 \%$ \\
\hline
\end{tabular}

Table-4MRI findings in preterm neonates.

\section{Distribution Of Cases According To Symptom Complex}

In this study $(n=100)$, maximum cases were of developmental delay (32). The other symptom complexes with which patients presented were seizures, cerebral palsy, hypotonia and drowsiness.

\begin{tabular}{|l|l|l|l|}
\hline Sr. No. & Pathology & No. of cases & Percentage \\
\hline 1) & Developmental delay & 32 & $32 \%$ \\
\hline 2) & Seizures & 28 & $28 \%$ \\
\hline 3) & Cerebral palsy & 20 & $20 \%$ \\
\hline 4) & Hypotonia & 14 & $14 \%$ \\
\hline 5) & Drowsiness & 6 & $6 \%$ \\
\hline & & Total $=100$ & \\
\hline
\end{tabular}

Table-5Distribution of cases according to symptom complex

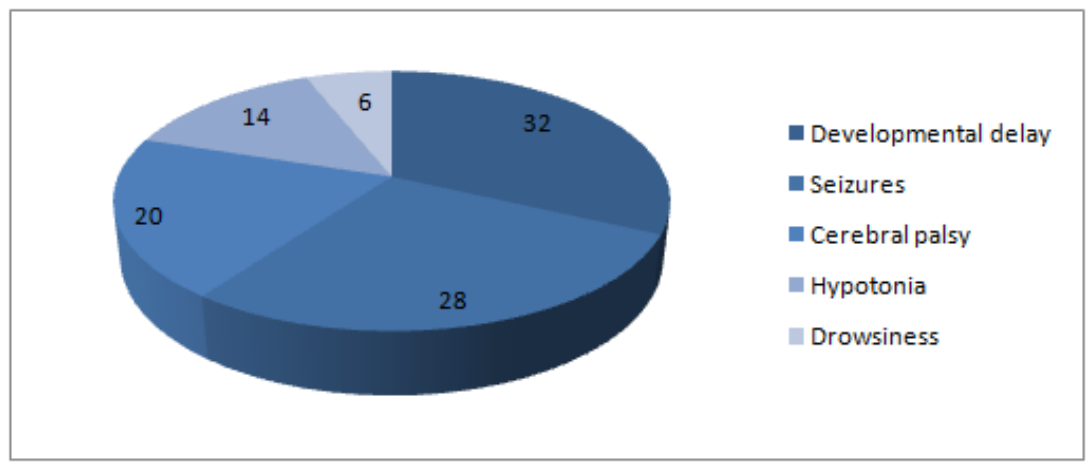

Figure-5Distribution of cases according to symptom complex 
Mri Brain in Perinatal Hypoxia - A Case Series.

\section{Distribution of Findings on MRI}

Out of 72 cases of HIE findings in perinatal hypoxia, maximum number of cases showed generalized cerebral atrophy $(61 \%)$, followed by T2WI hyperintensities. The other findings in preterm as well as term neonates were cerebellar atrophy, cystic encephalomalacia, delayed myelination, corpus callosum thinning, acute infarcts.

\begin{tabular}{|l|l|l|l|}
\hline \multicolumn{1}{|l|}{ Sr. No. } & Pathology & No. of cases $(\mathbf{n}=\mathbf{7 2})$ & Percentage \\
\hline 1) & T2 hyperintensity & 36 & $50 \%$ \\
\hline 2) & Cerebral atrophy & 44 & $61 \%$ \\
\hline 3) & Cystic encephalomalacia & 32 & $44 \%$ \\
\hline 4) & Delayed myelination & 28 & $39 \%$ \\
\hline 5) & Corpus callosum thinning & 24 & $33 \%$ \\
\hline $\mathbf{6 )}$ & Acute Infarcts & 14 & $19 \%$ \\
\hline 7) & Cerebellar atrophy & 12 & $17 \%$ \\
\hline 8) & Periventricular leukomalacia & 12 & $17 \%$ \\
\hline 9) & Germinal matrix hemorrhage & 2 & $0.3 \%$ \\
\hline
\end{tabular}

Table 6- Distribution of MRI findings.

Distribution of Findings on MRI According to Areas of Brain Involved:

\begin{tabular}{|l|l|l|l|l|}
\hline Sr. No. & Site & Preterm/Term & MRI Findings & No. of patients \\
\hline 1. & Cortex & Term & $\begin{array}{l}\text {-High signal intensity on T2 and FLAIR } \\
- \text { Infarct }\end{array}$ & $\begin{array}{l}12 \\
6 \\
\\
\end{array}$ \\
& & & - Atrophy & 32 \\
& & Preterm & - trophy & 12 \\
\hline 2. & Subcortical white matter & Term & -High signal intensity on T2 and FLAIR & 20 \\
\hline 3. & Periventricular White matter & Preterm & -High signal intensity on T2 and FLAIR & 12 \\
\hline 4. & Basal ganglia and thalami & Preterm & -High signal intensity on T2 and FLAIR & 4 \\
& & Term & -Infarct & 3 \\
\hline 5. & Hippocampus & Term & -High signal intensity on T2 and IR & 4 \\
\hline 6. & Cerebellum & Preterm & -High signal intensity on T2 and FLAIR & 3 \\
\hline 7. & Insula & Term & -Atrophy & 2 \\
\hline
\end{tabular}

Table 7- Distribution of findings on MRI according to areas of brain involved

\section{Distribution of Findings on MRI in Patients with Cerebral Palsy}

Out of 100 cases of perinatal hypoxia, 20 cases presented with cerebral palsy. Detailed analysis of MRI findings in these 20 cases is done as follows. $6(30 \%)$ cases were preterm infants and $14(70 \%)$ cases were term infants. Periventricular leukomalacia was the commonest finding in preterm infants, whereas, hyperintense lesions on T2WI images was the most common findings in term infants. The rest of the findings are given in the table below.

\begin{tabular}{|c|c|c|c|c|}
\hline Case No. & Age Sex & Pretermiterm & CP Type & MR Findings \\
\hline 1 & 9 Mnths M & Preterm & Diplegia & PVL Cerebral Atrophy \\
\hline 2 & $1 \mathrm{yz} / \mathrm{M}$ & Preterm & Diplega & PVL, Delayed Myelination \\
\hline 3 & $1 \mathrm{sr} / \mathrm{M}$ & Pretermi & Quadriplegia & PVL Cerebral Atrophy \\
\hline 4 & 4 menths $\mathrm{F}$ & Pteterm & Diplegia & Delayed Myelination, Corpus callosum thinsung \\
\hline 5 & 1 mnth $F$ & Preterm & Hemiplepin & Infact \\
\hline 6 & Franths $M$ & Preterm & Diplega & Normal \\
\hline 7 & $35 \mathrm{ys} \mathrm{M}$ & Term & Diplegia & Cerebral Atropby, T2HI \\
\hline 8 & 6 minths $F$ & Tenm & Diplega & Cerebral Atropby, T2HI \\
\hline 9 & $3,55 F$ & Term & Diplegia & Cerebral Atropby, T2HI \\
\hline 10 & 5 mnthe $M$ & Term & Hemiplegi2. & Infarct, Cerebral Atropby, T2HI \\
\hline 11 & 1 mnth $\mathrm{M}$ & Term & Quadriplepis & Cerebral Atroplay, T2HI Delayed Myelisation, Corpus callorum Thinning \\
\hline 12 & 3manthe: $M$ & Term & Dipleşa & Cerebral Atropby, T2HI Delayed Myelination. Corpus callosum Thinning \\
\hline 13 & $4 \mathrm{yrs} \mathrm{M}$ & Term & Quadriplegia & Global cystic encephalomalacia Corpus callosum Thinning \\
\hline 14 & $2 \mathrm{yrs} \mathrm{M}$ & Term & Diplegia & T2HI, Delaved Myelination \\
\hline 15 & $1 \mathrm{grF}$ & Term & Quadriplegia & Infarct T2HI Delayed Myelination \\
\hline 16 & $4 \mathrm{yrgF}$ & Tem & Hemiplegia & Infarct \\
\hline 17 & $5 \mathrm{yrs} / \mathrm{M}$ & Termi & Hemiplegia & Infurct \\
\hline 18 & $7 \mathrm{yrs} / \mathrm{M}$ & Termi & Hemiplepia & Normal \\
\hline 19 & $19 \mathrm{x} \mathrm{M}$ & Term & Diplegia & Normal \\
\hline 20 & 8 minths $F$ & Term & Diplepis & Nomal \\
\hline
\end{tabular}

Table 8- Distribution of findings on MRI in patients with cerebral palsy.

\section{Patterns of Hypoxic Ischemic Injury in Term Infants with Acute Presentation}

Out of 64 patients with a history of term delivery, infants presented with acute presentation were 10 in number. 5 main patterns of distribution of acute ischemic injury have been recognized. Of them, most showed a watershed predominant pattern. 
Mri Brain in Perinatal Hypoxia - A Case Series.

\begin{tabular}{|l|l|l|}
\hline Sr.No. & Pattern & No. of patients $(\mathrm{n}=10)$ \\
\hline $\mathbf{1}$ & Basal ganglia thalamus pattern (BGT) & 2 \\
\hline $\mathbf{2}$ & Watershed predominant pattern of injury (WS) & 4 \\
\hline $\mathbf{3}$ & White cerebrum pattern & 1 \\
\hline $\mathbf{4}$ & Periventricular white matter pattern & 2 \\
\hline $\mathbf{5}$ & Perinatal arterial ischaemic stroke (PAIS) & 1 \\
\hline
\end{tabular}

Table9- Distribution of patterns of hypoxic ischemic injury in term infants.

\section{Patterns of Hypoxic Ischemic Injury in Patients with Acute Presentation}

Of the total 100 patients, infants that presented with acute presentation with a history of a term delivery were 10 in number and those with a history of preterm delivery were 4 in number. Risk factors, HIE grades and MRI findings in 14 patients of acute presentation of HIE are analyzed as follows.

\begin{tabular}{|l|l|l|l|l|}
\hline Sr. No. & GA & $\begin{array}{l}\text { HIE Grade } \\
\text { (Sarnat and } \\
\text { Sarnat) }\end{array}$ & Risk factors & MRI Findings (n=14) \\
\hline 1 & Preterm & I & Idiopathic & Periventricular white matter pattern \\
\hline 2 & Preterm & II & PROM & Periventricular white matter pattern \\
\hline 3 & Preterm & III & Pre-eclampsia & $\begin{array}{l}\text { Basal ganglia- Thalami and Periventricular } \\
\text { white matter involvement with }\end{array}$ \\
\hline 4 & Preterm & III & Assessed Deliveries & Basal ganglia- Thalami involvement \\
\hline 5 & Term & I & Idiopathic & WM lesions \\
\hline 6 & Term & I & Idiopathic & WS predominat injury \\
\hline 7 & Term & I & Pre-eclampsia & WM lesions \\
\hline 8 & Term & I & Anemia & PLIC, WS predominat injury \\
\hline 9 & Term & II & Idiopathic & PLIC, WS predominat injury \\
\hline 10 & Term & II & Pre-eclampsia & WM lesions \\
\hline 11 & Term & II & Anemia & PAIS \\
\hline 12 & Term & II & Antepartum Hemorrhage & WS predominat injury \\
\hline 13 & Term & III & Pre-eclampsia & Basal ganglia- Thalami involvement \\
\hline 14 & Term & III & Antepartum Hemorrhage & Basal ganglia- Thalami involvement \\
\hline
\end{tabular}

Table10- Patterns of hypoxic ischemic injury in patients with acute presentation.

\section{Discussion}

Hypoxic-ischemic encephalopathy (HIE) in neonates is an important cause of mortality and morbidity and neurodevelopmental delay worldwide. It can lead to permanent brain damage and can also cause damage to other tissues of the body.

Almost $40 \%$ of deaths in children under the age 5 years occur in the neonatal period. Decrease in the mortality rate due to HIE observed in the developed countries could be due to the improved neonatal care, however the mortality and morbidity due to HIE in the developing countries still remains high and is a challenge. The total neonatal deaths occurring in the world due to non-specific conditions of HIE vary from 0.7 million7 to 1.6 million per year. In children affected by severe hypoxia, the mortality rate is reportedly $25-50 \%$. The maximum number of deaths occur in the first week of life as a result of multi- system failure or inadequate care. The children having severe neurologic deficit die in their infancy from aspiration pneumonia or various infections. Incidence of prenatal asphyxia is about 3.3\% in India and is usually related to gestational age and birth weight. It occurs in $9 \%$ of infants less than 36 weeks gestational age and in $0.5 \%$ of infants more than 36 weeks of gestational age accounting for $20 \%$ of perinatal deaths (or as high as $50 \%$ deaths if still births are included). ${ }^{[1]}$

Treatment for children suffered with hypoxic-ischemic encephalopathy was limited to supportive care for a long time, but now recent advances for effective therapies have been developed. It is a treatable problem and early identification and intervention is necessary to prevent the long term brain damage.Advances in MRI technique have made excellent progress over the last few years.MRI and MRS with the help of DTI is useful in detection of exact patterns of early and late damage in infants with HIE.

Both terminal zones of myelination and perivntricularleukomalacia appear hyperintense on T2WI and FLAIR and their differentiation is extremely difficult. Terminal zones are the persistent areas of high signal intensity in the white matter lateral to the bodies of the lateral ventricles and in the dorsal and superior to the ventricular trigones on T2-weighted images. The difference is best elicited on coronal T2WI and FLAIR images. The periventricular leukomalacia lesions are more sharply defined. They are present more inferiorly; lateral to the trigones and near the optic radiations. They are typically brighter on T2WI and FLAIR sequences than terminal zones of myelination. Periventricular leukomalacia is associated with loss of brain tissue, which results in the irregularity of the ventricular wall, abnormally deep cortical sulci, sometimes extending down to the ventricular surface, and thinning of the body of the corpus callosum. A layer of myelinated white matter is present between the trigone of the ventricle and the terminal zones in normal patients. The very high signal 
intensity of the peritrigonal areas compared to surrounding white matter on FLAIR images with the presence of local atrophy favor PVL. ${ }^{[2]}$

Differentiation of hypoxic-ischemic cerebral injury from normal myelination is important for prediction of neurologic development. T1-weighted images help distinction of infants with hypoxic- ischemic brain damage from those with normal myelination. The posterior limb of the internal capsule (PLIC) is myelinated at birth and appears hyperintense on T1-weighted images. The loss of this normal high signal intensity in the PLIC in infants with HIE, indicates a delay in myelination or insult to previously myelinated tracts. This loss of high SI in the PLIC, though sometimes subtle, is associated with unfavourable outcome. ${ }^{[3]}$

\section{Patterns of HIE in preterm and term neonates:}

An ischemic event lasting for more than 10 minutes is needed to induce parenchymal changes. The extent of injury increases with prolonged duration of the insult. Thus the patterns of injury in infants can be divided into term and preterms depending upon gestational age and being mild to moderate and severe hypoperfusion injuries, depending upon the severity of insult. ${ }^{[4]}$

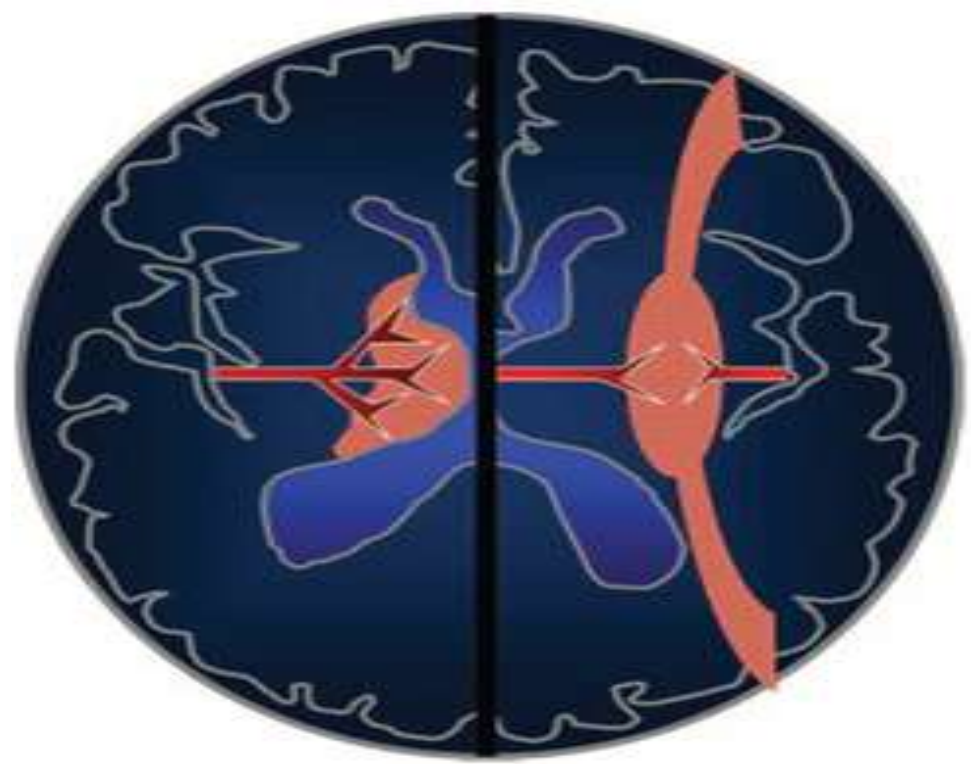

Figure- 6 Patterns of brain injury in mild to moderate hypoperfusion. Schematic of the premature neonatal brain (left) and that of the term infant (right) illustrates how the vascular supply changes with maturation and affects the pattern of brain injury in HIE. The premature neonatal brain (left) has a ventriculopetal vascular pattern, and hypoperfusion results in a periventricular border zone (red shaded area) of white matter injury. In the term infant (right), a ventriculofugal vascular pattern develops as the brain matures, and the border zone during hypoperfusion is more peripheral (red shaded area) with subcortical white matter and parasagittal cortical injury.

(Source- Christeine P. Chao et al. Neonatal Hypoxia Ischemic Encephalopathy: Multimodality Imaging. Radiographics 2006; 26:159-172. ${ }^{[5]}$ )

\begin{tabular}{|l|l|l|}
\hline Age of Child & $\begin{array}{l}\text { Mild to Moderate } \\
\text { Hypotension }\end{array}$ & Profound Hypotension \\
\hline $\begin{array}{l}\text { Premature neonate (up to } 34 \\
\text { postconceptional weeks) }\end{array}$ & $\begin{array}{l}\text { Periventricular } \\
\text { white matter injury }\end{array}$ & Thalamic, basal ganglia, and brainstem injury \\
\hline $\begin{array}{l}\text { Term neonate ( 36 to } ~ 56 \\
\text { postconceptional weeks) }\end{array}$ & $\begin{array}{l}\text { Parasagittal } \\
\text { watershed injury }\end{array}$ & $\begin{array}{l}\text { Dorsal brainstem, ventral cerebellar vermis, thalamus, } \\
\text { basal ganglia and perirolandic cortex injury }\end{array}$ \\
\hline $\begin{array}{l}\text { Older child (more than } 4 \text { to } \\
6 \text { postnatal months) }\end{array}$ & $\begin{array}{l}\text { Parasagittal } \\
\text { watershed injury }\end{array}$ & Basal ganglia and diffuse cortical injury \\
\hline
\end{tabular}

Table-11 Patterns Of Injury In Diffuse Hypoxic-Ischemic Injury

Linda S. de Vries\&FlorisGroenendaal in 2014 identified four patterns of acute infarcts in term neonate. ${ }^{[6]}$

\section{1) Basal ganglia pattern:}

It affects bilateral central grey nuclei (ventro lateral thalami and posterior putamina) and perirolandic cortex. It is generally seen following an acute event, such as a ruptured uterus, placental abruption or umbilical cord prolapse, and is also referred to as a pattern following 'acute near total asphyxia. The absence of a normal 
high-signal intensity of the posterior limb of the internal capsule (PLIC) on T1WI is highly predictive of severe adverse sequelae. On spin echo MRI sequences, inversion of the signal within the PLIC can be seen from 48 to $72 \mathrm{~h}$ onwards. DWI will show early changes in the basal ganglia/thalami. Children with the BGT pattern of injury tend to be severely disabled due to dyskinetic cerebral palsy (CP).

\section{2) Watershed predominant pattern of injury (WS)}

$t$ is the type of pattern that can be seen after 'prolonged partial asphyxia'. The vascular watershed zones (anterior-middle cerebral artery and posterior-middle cerebral artery) are involved, affecting white matter and in case of more severe insult also the overlying cortex. The lesions can be uni- or bilateral, posterior and/or anterior. On spin echo MR images loss of the cortical ribbon and thus the grey-white matter differentiation can be seen. DWI is however helpful in making an early diagnosis. A follow up MRI may show cystic evolution with or without atrophy and gliotic changes. This type of insult is usually mild and thus the onset of neurological signs can be delayed. Severe motor impairment is uncommon in this. Symptomatic parietooccipital epilepsy may occur later in childhood, often associated with reduced intelligence quotients and visuospatial cognitive function.

\section{3) White cerebrum pattern}

Marked involvement of the subcortical white matter and cortex is noted with relative sparing of the immediate periventricular white matter and central grey matter. This is referred to as the 'white cerebrum', as DWI shows completely white cerebrum, contrasted to a normal looking cerebellum. This condition tends to be fatal, but in case of survival, muticysticencephalomalacia eventually develops.

\section{4) Periventricular white matter pattern.}

It is similar to the punctate white matter lesions in the preterm infant. It is associated with a mild degree of encephalopathy and fewer clinical seizures. Thispattern of brain injury is observed in newborn infants with congenital heart defects.

\section{5) Perinatal arterial ischemic stroke (PAIS), perinatal haemorrhagic stroke (PHS) and sinovenous thrombosis.}

It is seen in newborns presenting with encephalopathy and/or seizures. Restricted diffusion at the level of the internal capsule and the middle part of the cerebral peduncle, referred to as 'pre-Wallerian degeneration' can be appreciated. It is then followed by Wallerian degeneration at 6-12 weeks and beyond. Presence of Wallerian degeneration at birth suggests an antenatal onset of the insult.

We carried out a study on 100 patients of perinatal hypoxia presenting with various symptom complexes. Of them, $72 \%$ (72) were abnormal and $28 \%$ (28) were normal studies. Our study correlates with study carried out by M.A. Rutherford. ${ }^{[7]}$ Our findings correlate well with these studies.

In our study of 100 patients; $56 \%$ (56) were males and $46 \%$ (46) were females with a mean age group of 6 years (age range $=0$ to 12 years). Maximum patients were of age group 1 to 5 years. In a study carried out by AzharMunir Qureshi et al; $79.6 \%$ were males and $20.4 \%$ were females. ${ }^{[1]}$ In another study done by D.J.A. Connolly, the age group range taken into consideration was 1 to 24 years. Of them maximum patients were between the age group of 1 to 5 years. ${ }^{[8]}$ Thus, our findings corroborate well this study.

In our study $36(36 \%)$ patients were preterms and $64(64 \%)$ had term delivery. These findings are in accordance with study performed by R Yin. He carried out the study on 42 patients in whom 12 were premature (28.5\%) and 30 were full-term (71. 4\%). ${ }^{[9]}$ In another study done by AzharMunir Qureshi et al on 181 infants, out of 181 neonates $77.9 \%$ were full term, $19.1 \%$ were premature. Thus, overall, our findings correlate with these studies. ${ }^{[1]}$

Various risk factors for HIE have been proposed over the years. In our study, PIH was commonest risk factor $20 \%$ (20) followed by anaemia $15 \%(15)$. The other risk factors were placental factors $10 \%(10)$, perinatal infections $8 \%$ (8), and assessed delivery 7\% (7). No risk factors were identified in $25 \%$ (25) cases. Caesarean section was done in $15 \%$ (15) of the mothers. In the study carried out by AzharMunir Qureshi et al on 181 patients; the most common risk factor was PIH, observed in $27.7 \%$ of the mothers, followed by anaemia seen in $16 \%$. Placental causes were present in $18.3 \%$. Assessed delivery was done for $7.2 \%$ of the patients and in $15.5 \%$ no maternal cause was found. $34.3 \%$ were delivered by Caesarean section. ${ }^{[1]}$ Thus, our findings corroborate well with this study.

Inclusion of antenatal examination, home delivery, hospital delivery was taken into consideration in our study. We found that around $55 \%$ (55)cases were primigravida. Of them, around $80 \%$ (44) patients had not undergone any antenatal examination. Rest $20 \%$ (11) had undergone antenatal examination. Home delivery was done in $27.2 \%$ (15) of primigravida mothers, whereas hospital deliveries were done in $72.8 \%$ (40). In the remaining $45 \%$ of multigravida, $75 \%$ (33) patients did not undergo any antenatal checkup and the rest of the 25 
$\%$ (12) underwent antenatal examination. $31.1 \%$ (14) of multigravida had undergone home deliveries.Rest of the $68.9 \%$ (31) underwent hospital deliveries. In the study done by AzharMunir Qureshi et al52.5\% of the mothers were primigravidaand of them 5\% were managed at home. $47.5 \%$ cases were multigravida and of them, $8 \%$ were home deliveries. Thus my study is in accordance with this study. ${ }^{[1]}$

We evaluated $64(72 \%)$ patients with a history of term delivery. Out of which $28.1 \%$ (18) were normal studies and $71.8 \%$ (46) cases were abnormal. Out of the abnormal cases cortical and subcortical T2 hyperintensities were noted in 60\% (36) cases. Cerebral atrophy was also common and found in $50 \%$ (32) cases. In patients presenting with acute symptoms, $15.6 \%$ (10) cases showed acute infarcts. Delayed myelination was seen in $31.2 \%$ (20) of the patients. Mary Rutherford with his colleagues performed a study on patients with HIE and tried to find the correlation of MR findings with clinical outcome. He found cortical and subcortical T2 hyperintensities in $50 \%$ of the patients and cerebral atrophy in $50 \%$ of the cases. Basal ganglia infarction was observed in $18 \%$ cases and $6 \%$ cases showed insular infarcts. Delayed myelination was present in $50 \%$ of the infants. ${ }^{[10]}$ The difference in the results of our study and other studies can be because of difference in the sample size.

We studied 36 (28\%) patients who were born with the history of preterm delivery. Of these, $72.3 \%$ cases were found to be abnormal. 33.3\% (12) cases showed periventricular leukomalacia and $33.3 \%$ (12) patients showed cerebral atrophy as the commonest findings. Delayed myelination and corpus callosum thinning was observed in $22.2 \%$ (8) cases. $27.7 \%$ cases did not show any significant abnormality on MRI. Acute infarcts were seen in $11.1 \%$ (4) cases. Cerebellar atrophy was present in $5.5 \%$ (2) cases. Germinal matrix hemorrhage was diagnosed in $5.5 \%$ (2) cases. In a study carried out by Gul Serdaroglu et al, 89 children with PVL were evaluated. The aim of this study was to find out neurodevelopmental delay in children with periventricular leukomalacia (PVL). PVL was divided into 3 grades: grade I, unilateral or bilateral areas of periventricular hyperintensity; grade II, hyperintensity more than 3; grade III, hyperintense lesions more than 3 and ventricular wall irregularity; grade IV, diffuse PVL and ventricular dilatation. Thinning of the corpus callosum and cortical atrophy was identified respectively in $73 \%$ and $47.2 \%$ of the patients. Delayed myelination was noted in $14.3 \%$ cases. MRI was normal in $18 \%$ of the infants. ${ }^{[11]}$ In a similar study done by PavithraLogitharajah, the major sites of injury were basal ganglia (BG, 75\%), white matter (89\%), Cortex was involved $(58 \%)$ followed by brain stem in $44 \%$ cases No abnormality was found in $32 \%$. Significant central gray matter and brainstem injury was found in many preterm infants with HIE. Neonatal MRI findings allowed accurate prediction of neurodevelopmental outcome on follow up studies. ${ }^{[12]}$ The difference in the results of our study and other studies can be because of different age selection criteria of our study compared to other studies.

We came across various symptom complexes with which patients presented. Of them, maximum patients presented with developmental delay 32\% (32). This was followed by epilepsy seen in $28 \%$ (28) patients. Cerebral palsy was seen in $20 \%$ (20) cases. Patients presenting with hypotonia were $14 \%$ (14) whereas $6 \%$ (6) cases had an acute presentation in the form of drowsiness or altered sensorium. Maximum cases of developmental delay were noted in the study performed by Gul Serdaroglu et al. Epilepsy was found in $33.7 \%$ and cerebral palsy in $30.8 \%$ of the patients. Approximately $28 \%$ patients had diplegia. ${ }^{[11]}$ Thus, our findings are in concordance with this study.

In our study, 20 patients presented with cerebral palsy. Out of these 20 cases $30 \%$ (6) cases were preterm infants. The most common finding that we encountered was periventricular leukomalacia seen in $50 \%$ (3) of the cases. It was followed by cerebral atrophy and delayed myelination which was seen in $33.3 \%$ (2) cases. Corpus callosum thinning and infarcts were seen in $16.6 \%$ (1) patients. Whereas $16.6 \%$ (1) cases did not show any abnormality. $70 \%$ (14) cases of term infants presenting with cerebral palsy were studied. The commonest finding in term infants was hyperintense lesions on T2WI images seen in 64\% (8) cases. The next common finding was cerebral atrophy, which was present in $50 \%$ of the cases (7). Delayed myelination and acute infarcts were noted in $28.5 \%$ (4) cases. The less common finding was corpus callosum thinning seen in $21.4 \%$ (3) cases. No abnormality was seen in $21.4 \%$ (3) of the cases. Our findings were similar to those studied by R.Yin. He performed a study of MRI findings in 42 patients, of which 8 were premature (38\%), 13 were full-terms $(62 \%)$. Periventricular leukomalacia was seen in $66.6 \%$. Cerebral atrophy was seen in $33.3 \%$ cases and $33.3 \%$ children did not demonstrate PVL. The other group he studied included 30 children who were born between 37 and 42 weeks gestation that is term infants. The MRI findings demonstrated T2 hyperintensities as the most common finding seen in approx. $30 \%$ patients. It was followed by cerebral atrophy seen in $20 \%$ cases and other lesions in $20 \%$ cases. Acute infacrts were noted in $13 \%$ of the patients. Delayed myelination was seen in $7 \%$ cases and corpus callosum thinning in $6 \% .{ }^{[9]}$ Charles L. Truwit did a study on 40 patients with cerebral palsy. Of these, 11 were premature and 29 were term infants. Of the 11 patients born prematurely, MR revealed deep white matter loss, especially in the peritrigonal regions. $81 \%$ of the scans demonstrated thinning of the corpus callosum. No abnormalities were noted in the basal ganglia or thalami. $27 \%$ had diminished caliber of the brainstem. MR was done in 29 patients born at term, out of which $66 \%$ had diminished deep white matter. $6 \%$ had delayed myelination. In $55 \%$, the corpus callosum was thinned either focally (involving the 
posterior body and/or splenium) or diffusely. Ventricular abnormalities were seen in $41 \%$ with an irregular ventricular contour and ventricular enlargement and cerebral atrophy. Cortical thinning was seen in $10 \%$ term patients, one of whom had findings diagnostic of multicysticencephalomalacia.20\% cases had basal ganglia and/or thalami involvement. ${ }^{[13]}$ In another case series, RubaBenini studied 126 patients with cerebral palsy. Of these, $71 \%$ had abnormal findings and 36 had normal brain scans. Compared with other CP types, normalappearing MRI was more prevalent in dyskinetic CP $72 \%$ and less prevalent in spastic hemiplegic $\mathrm{CP}$ $10 \%$.Thus, genetic or functional, rather than gross structural lesions, may underlie the pathophysiology of $\mathrm{CP}$. ${ }^{[14]}$ Overall our findings corroborate with both the studies.

We evaluated 5 patterns of distribution of acute ischemic brain injury in patients presenting with a history of term delivery. Of the 64 patients with a history of term delivery, $15.6 \%$ presented with acute symptoms. The watershed predominant injury pattern was the most common finding observed in $40 \%$ (4) of the patients. Basal ganglia- Thalami pattern and periventricular white matter pattern was noted in $20 \%$ (2) of the cases respectively. $10 \%$ cases showed a white cerebrum pattern and Perinatal Arterial Ischemic Stroke (PAIS) was seen in $10 \%$ cases.84Abnormal signal intensity appearing hypointese on T1WI images was noted in the posterior limb of internal capsule (PLIC) was noted in $20 \%$ (2) patients. This sign helped in differentiating the normal myelination from hypoxic injury. ${ }^{[3]}$

We also studied patterns of acute injury in $11.1 \%$ (4 out of 36) patients with a history of preterm delivery. Of them, $50 \%$ (2) had an abnormal signal intensity in the bilateral periventricular white matter. 50\% patients showed Basal ganglia -Thalami involvement.

An overall interpretation of patient characteristics and findings on the imaging when compared with the data available according to the review of literature corroborated very well. There are no significant and inexplicable differences in the range/spectrum of MRI findings in patients presented with HIE.

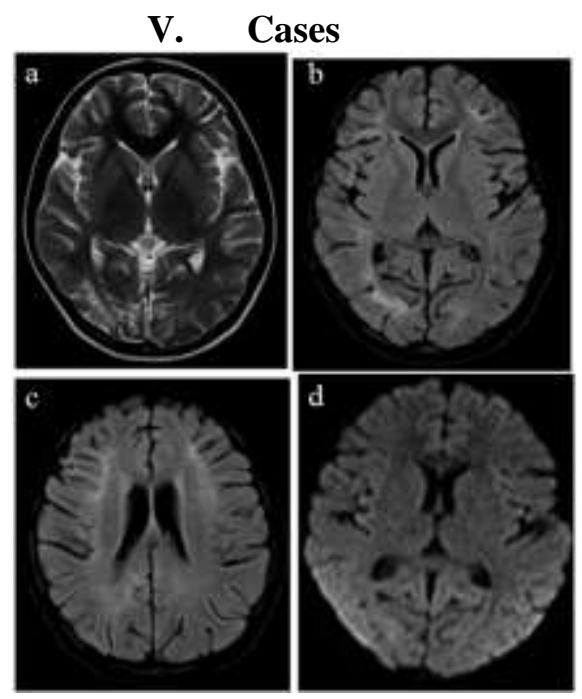

Figure -7 Axial MRI T2WI (a), FLAIR (b) and (c) show hyperintense areas in the bilateral periventricular white matter with no restricted diffusion on DWI (d) suggestive of HIE.

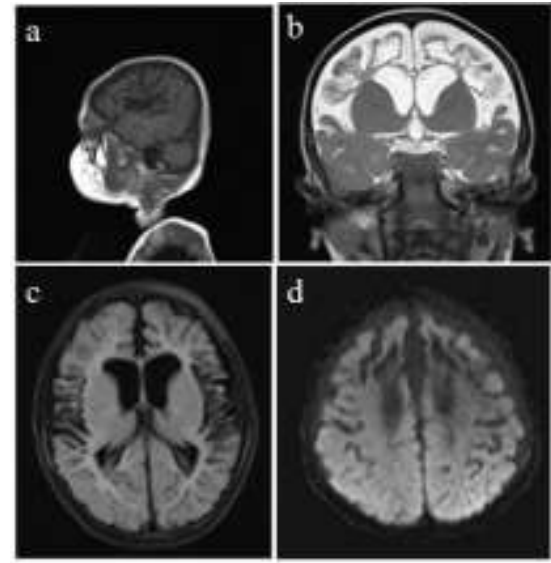

Figure-8 Altered signal is seen in bilateral high fronto-parietal region predominantly along cortex and subcortical white matter and also extending to periventricular white matter appear hypointense on T1WI (a) and 
hyperintense in T2WI (b) and FLAIR (c) with no evidence of restricted diffusion (d) suggestive of gliotic changes. Ex vacuo dilatation of both lateral ventricles is also seen.
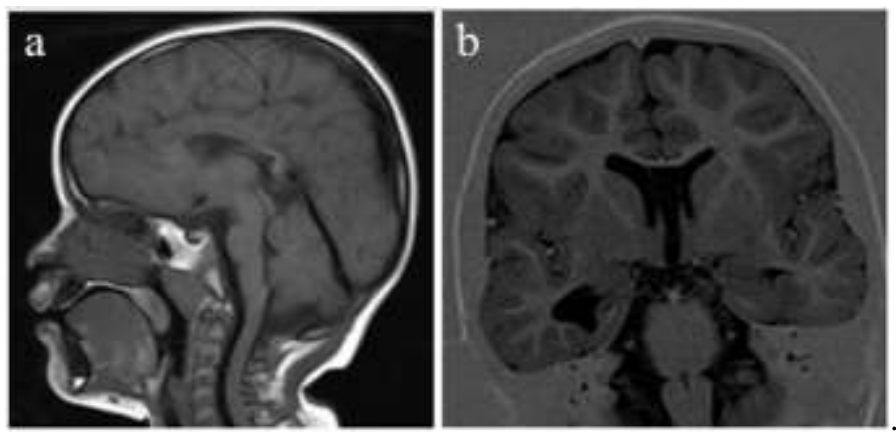

Figure-9 Midsagittal MR T1WI (a) and coronal 3D T1WI IR (b) images showing thinning of corpus callosum.

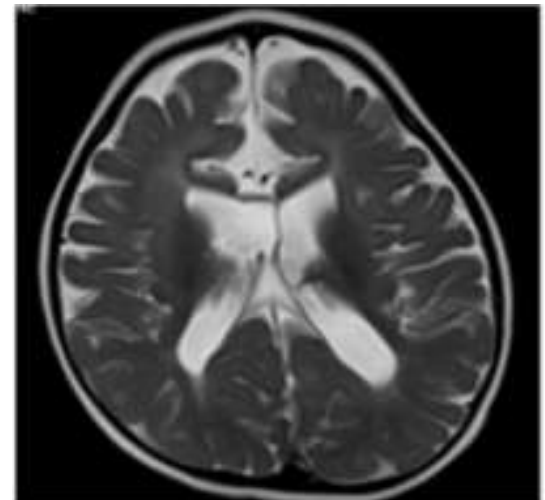

Figure 10 - Axial T2WI MR image showing delayed unmyelinated white matter appearing hyperintense in bifrontal region.
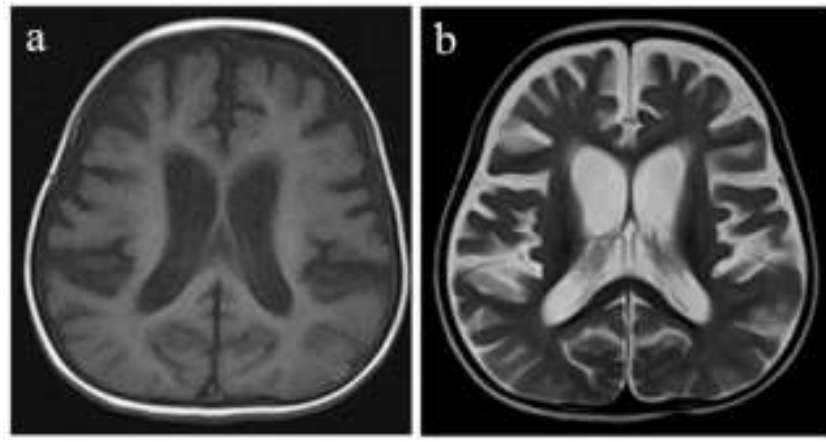

Figure -11 Axial T1WI (a) and T2WI (b) MR images showing prominence of ventricular system and cortical sulci suggestive of generalized cerebral atrophy.
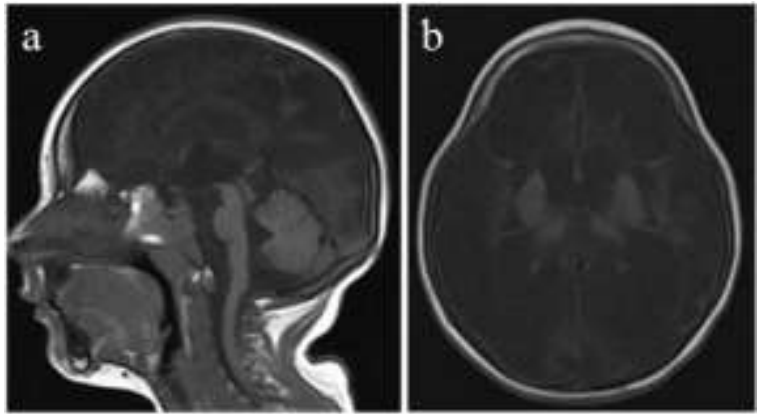

Figure - 12 Mid sagittal (a) and axial (b)T1WI MR at the level of basal ganglia showing changes of cystic encephalomalacia involving both the cerebral hemispheres showing CSF intensity. Note that the deep nuclei are spared. 

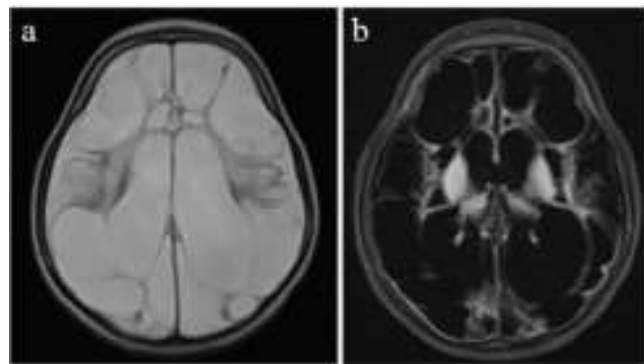

Figure - 13: Multiple areas of cystic encephalomalacia are noted in bilateral cerebral hemispheres appearing hyperintense on T2WI (a) and suppressed on FLAIR images (b). The basal ganglia and thalami are spared in this case, thus confirming that it is a term infant.
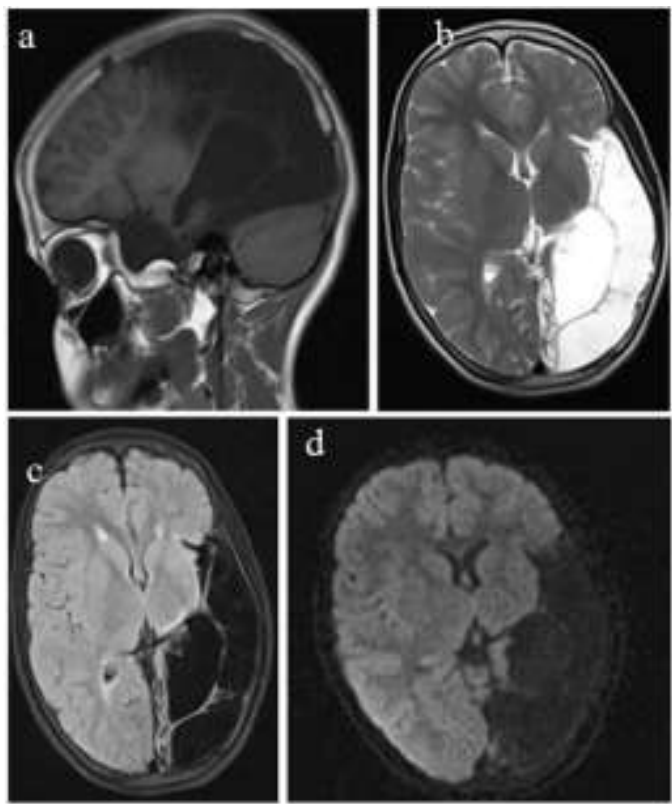

Figure - 14 Left para sagittal T1WI (a), axial T2WI (b), axial FLAIR (c) and axial DWI (d) MR images at the level of lateral ventricles showing area of cystic encephalomalacia involving the left MCA territory appearing hypointense on T1WI and FLAIR, hyperintense on T2WI images with no restriction on DWI.
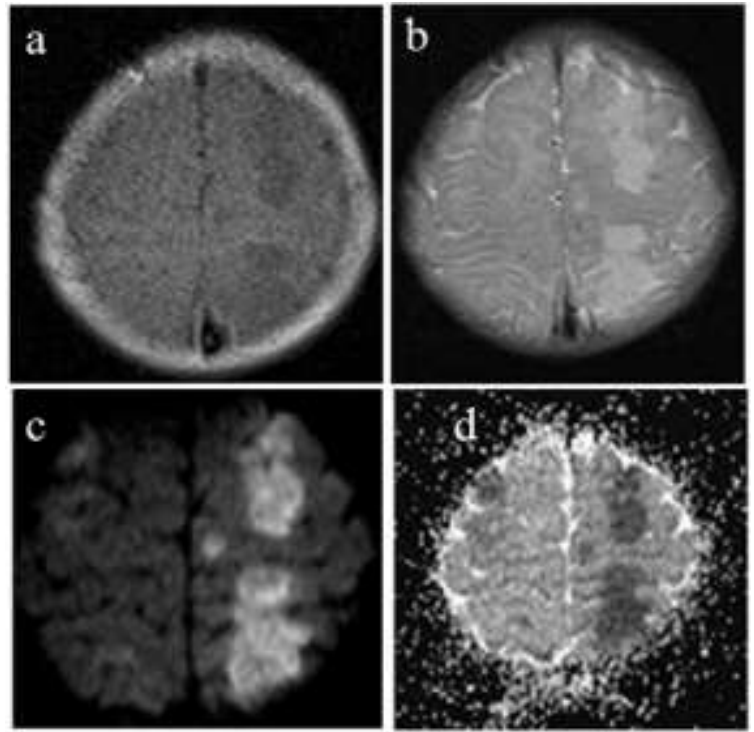

Figure -15 Axial T1WI (a) and T2WI (b) images showing areas of altered signal intensity in left watershed areas appearing hypointense on T1WI and hyperintense on T2WI images. These areas are showing restriction on

DWI (c) with corresponding low ADC values (d). This was suggestive of acute infarct in left anterior and posterior watershed areas suggestive of watershed pattern. 

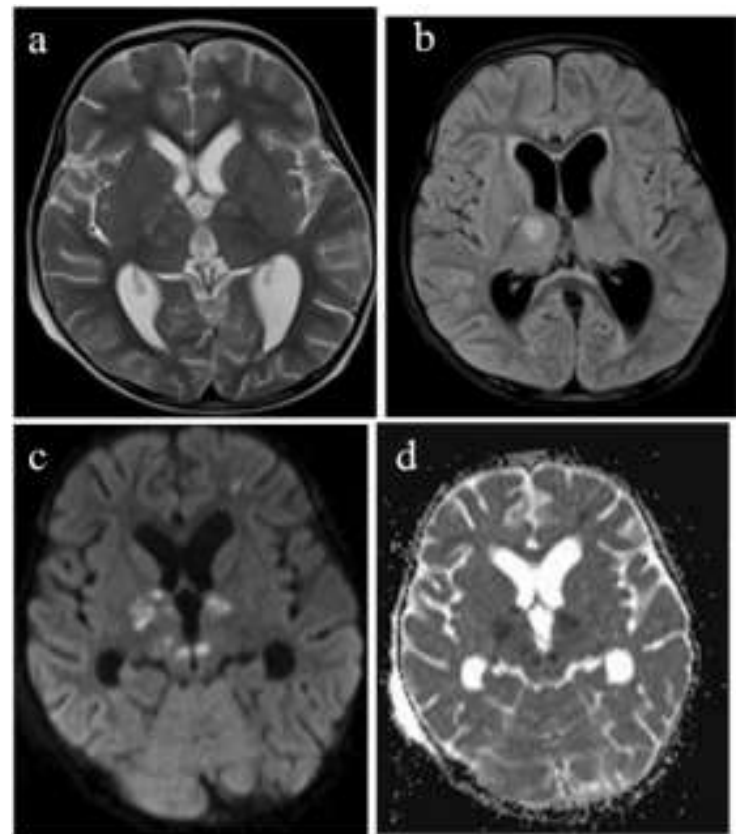

Figure - 16 Areas of altered signal intensity are seen involving deep nuclei in a preterm infant appearing hyperintense on T2WI (a) and FLAIR (b) images, showing restriction on DWI (c) with corresponding low ADC values (d) suggestive of acute infacrts with basal ganglia pattern.
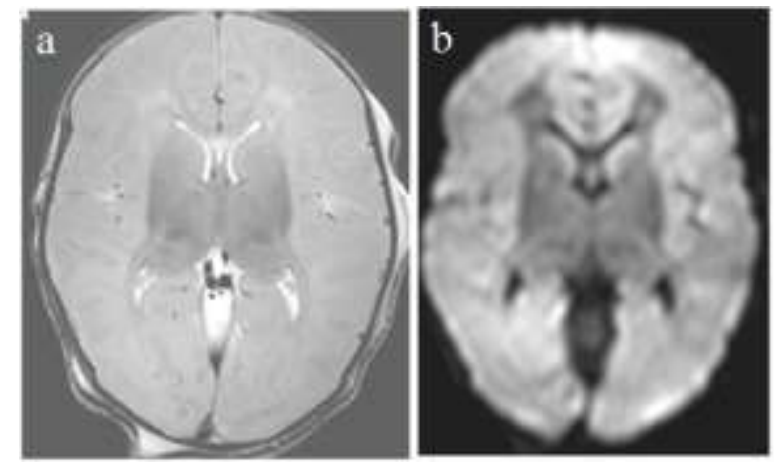

Figure -17 Axial MR T2WI (a) and DWI (b) images at the level of lateral ventricles show areas of altered signal intensity involving the cerebral white matter with relative sparing of the periventricular white matter and deep nuclei appearing diffusely hyperintense on T2WI images with corresponding restriction on DWI. This is suggestive of acute infarct with the white brain pattern of injury.

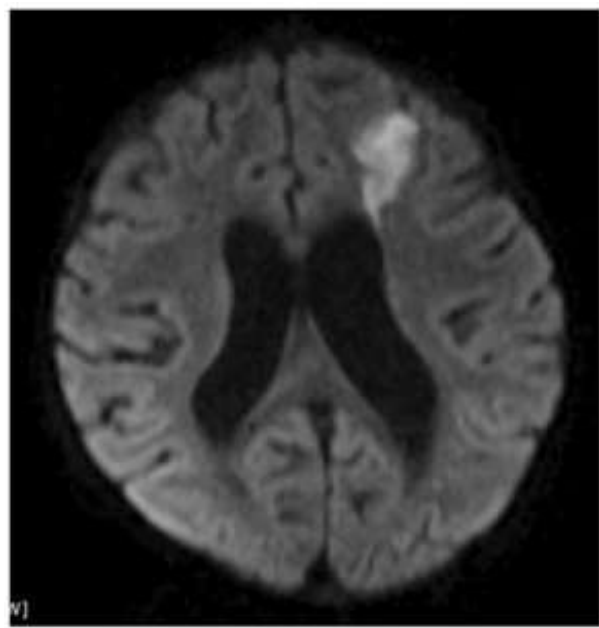

Figure - 18 Axial DW MR Image at the level of lateral ventricles showing focus of restricted diffusion in the left frontal periventricular white matter. This is suggestive of periventricular type of pattern of acute infarct. 

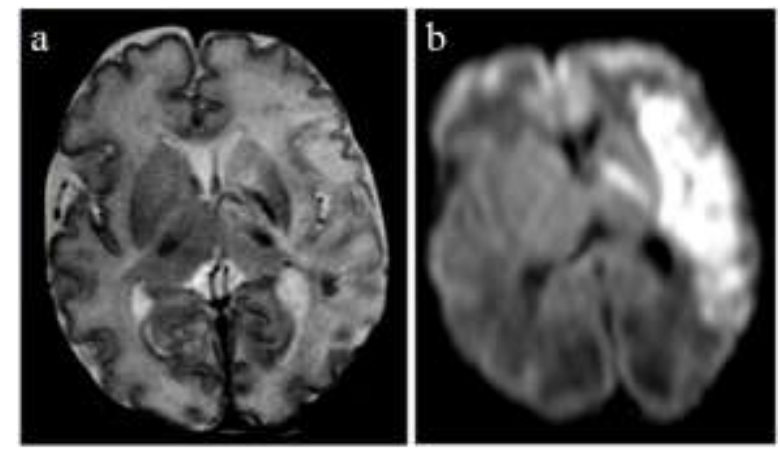

Figure-19 Axial T2WI (a) and DW (b) MR images at the level of lateral ventricles showing acute infarct involving left MCA territory appearing hyperintense on T2WI and showing restriction on DWI. This is the perinatal arterial ischemic stroke pattern.
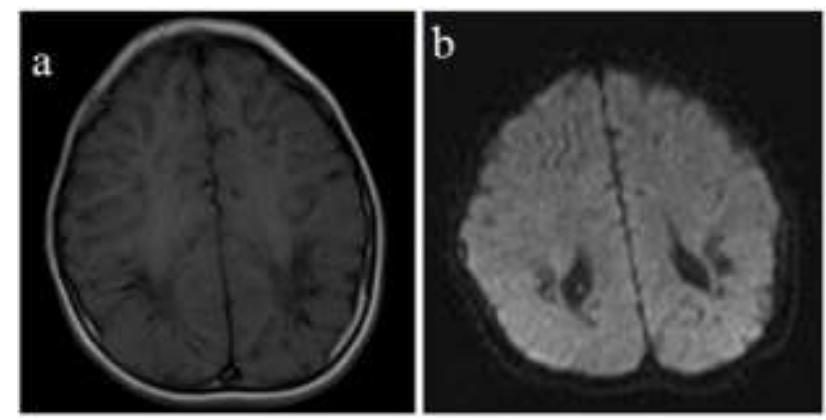

Figure -20 Axial MR images at the level of lateral ventricles showing areas of altered signal intensity in periventricular areas posteriorly appearing hypointense on T1WI (a). These areas do not show restriction on DWI (b) suggestive of HIE.
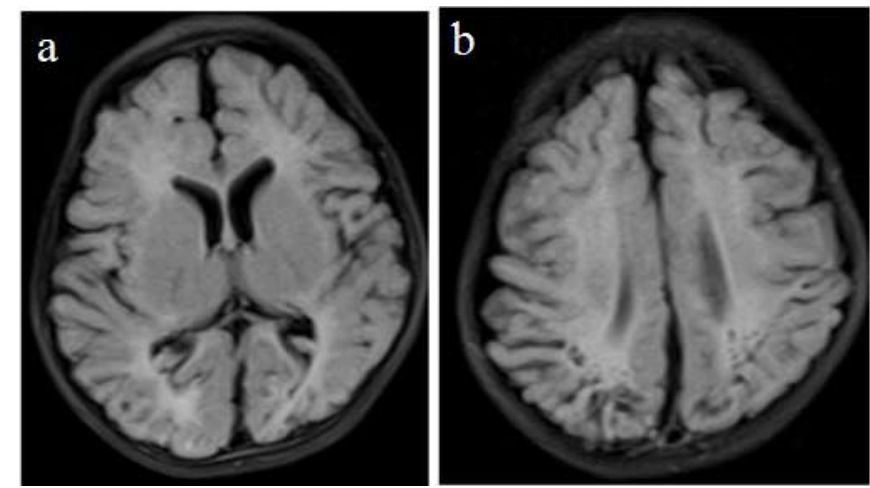

Figure -21 Axial FLAIR MR images at the level of body of lateral ventricles (a) and at the level of corona radiata (b) showing bilateral periventricular hyperintensities with reduced white matter in both occipital lobes (forceps major) suggestive of periventricular leukomalacia.

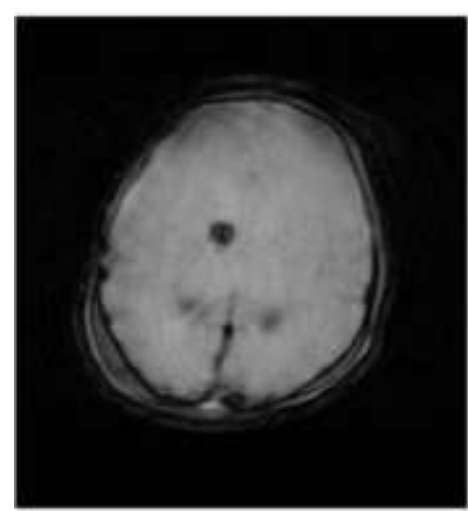

Figure - 22 Axial GRE image shows area of blooming in the right germinal matrix suggestive of germinal matrix haemorrhage. 

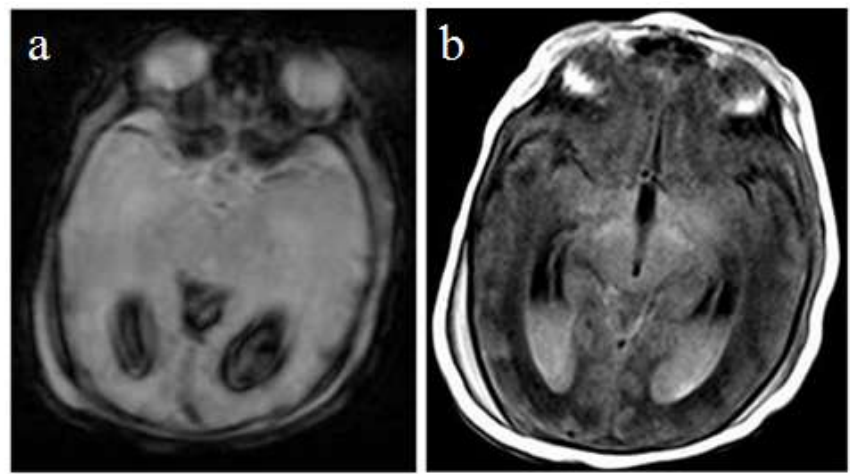

Figure - 23 Axial GRE (a) and T1WI (b) images show intraventricular hemorrhage in bilateral occipital horns of lateral ventricles following germinal matrix haemorrhage with early hydrocephalus, showing area of blooming on GRE images appearing hyperintense on T1WI images.

\section{Conclusion}

MRI was able to differentiate between patterns of brain injury, according to the brain maturity, severity and length of the ischemic insult. In our case series, patients presenting late with HIE were more than that of those presenting with acute presentation. This suggests that there is a lack of public health awareness as well as neonatologists in developing countries. Protocol for doing MRI in a suspected case of HIE needs to be formulated.

In our case series, serial follow up of patients suffered with perinatal hypoxia on MRI for the evolution of the lesions and neurodevelopmental outcome was not done due to time constrains. Also, MRI brain was normal in0 few patients who had suffered with perinatal hypoxia. This can be overcome by performing MRS within 2-3 days of insult in patients with normal conventional MRI brain studies. DTI can also be useful for detailed evaluation of the sequelae of HIE in whom conventional MRI brain studies were inconclusive.

Apart from this timely recognition of the risk factors and public health awareness needs to be created. Improvements in maternal health and regular antenatal checkups should be emphasized. Also, follow up MRI studies for those children who have suffered with perinatal hypoxia is extremely important to know the prognosis of the condition.

\section{References}

[1]. Qureshi AM, Anees R., Tahir S. S. Hypoxic ischemic encephalopathy in neonates. J Ayub Med Coll Abbottabad 2010; 22:4-9.

[2]. Baker LL, Stevenson DK, Enzmann DR. End stage periventricular leukomalacia: MR imaging evaluation. Radiology 1988; 168:809-815.

[3]. L. Liauw et al.Differentiating Normal Myelination from Hypoxic- Ischemic Encephalopathy on T1-Weighted MR Images: A New Approach. American Journal of Neuroradiology 2007; 28:660-5.

[4]. Suzuki J, Ito M. Coincidence patterns of cerebral palsy in Shiga Prefecture, Japan, 1977-1991. Brain Dev 2002; 24:39-48.

[5]. Christeine PC.et al Neonatal Hypoxic Ischemic Encephalopathy: Multi Modality Imaging Radio Graphics 2006; 26:S159-S172

[6]. Linda S. de Vries\&FlorisGroenendaal. Patterns of neonatal hypoxic-ischaemic brain injury. Neuroradiology 2010; 52:555-566

[7]. Rutherford M, Pennock J, Schwieso J, Cowan F, DubowitzL.Hypoxic-ischaemic encephalopathy: early and late magnetic resonance imaging findings in relation to outcome.Arch Dis Child Fetal Neonatal Ed. 1996 Nov;75(3):F145-51.PMID: 8976678.

[8]. Connolly DJ, Widjaja PD. Griffiths. Involvement of the anterior lobe of the cerebellar vermis in perinatal profound hypoxia. American Journal of Neuroradiology 2007; 28(1):16-9.

[9]. Yin R, Reddihough D, Ditchfield M, Collins KJ. Magnetic resonance imaging findings in cerebral palsy. J Paediatr Child Health 2000; 36: 139-144.

[10]. Mary R, Jacqueline P, Jane S, Frances C, Lilly D. Hypoxic ischemic encephalopathy: early and late Magnetic Resonance Imaging findings in relation to outcome. Archives of Disease in Childhood 1996; 75: 145-151

[11]. Serdaroglu G1, Tekgul H, Kitis O, Serdaroglu E, GokbenS.Correlative value of magnetic resonance imaging for neurodevelopmental outcome in periventricular leukomalacia. Dev Med Child Neurol 2004; 46:733-9.

[12]. Pavithra L, Rutherford M A, Frances M. C. Hypoxic-Ischemic Encephalopathy in Preterm Infants: Antecedent Factors, Brain Imaging, and Outcome. Pediatr Res 2009; 66:222-9.

[13]. Charles L. Truwit, Barkovich A J, Thomas K, Ferriero M. Cerebral Palsy: MR Findings in Patients. American Journal of Neuroradiology 1992; 13:67-78.

[14]. Benini R, Dagenais L, Michael I. S. Normal Imaging in Patients with Cerebral Palsy: What Does It Tell Us? J Pediatr 2013; 162:369-74 